\title{
Methodikbaukasten zur effizienten, zielgerichteten RDE-Entwicklung - Potenziale und Perspektiven
}

Hipp, Johannes; Schmidt, D.; Bauer, S. et al.

(2019)

DOI (TUprints):

Lizenz:

Publikationstyp:

Fachbereich:

Quelle des Originals: https://doi.org/10.25534/tuprints-00014571

lediglich die vom Gesetz vorgesehenen Nutzungsrechte gemäß UrhG

Konferenzveröffentlichung

16 Fachbereich Maschinenbau

https://tuprints.ulb.tu-darmstadt.de/14571 


\section{Methodikbaukasten zur effizienten, zielgerichteten RDE Entwicklung Potentiale und Perspektiven}

Johannes Hipp, Wissenschaftlicher Mitarbeiter,

D. Schmidt, S. Bauer, T. Steinhaus, Prof. Dr. C. Beidl,

Institut für Verbrennungskraftmaschinen und Fahrzeugantriebe (VKM), TU Darmstadt 
Potentiale und Perspektiven

\section{Motivation und Zielsetzung}

Die Umsetzung der aktuellen Emissionsgesetzgebung führt zu einem Paradigmenwechsel in der Entwicklung von Verbrennungsmotoren. Neben einer neuen Prüfprozedur (WLTP) inklusive neuem Prüfzyklus (WLTC) wurde zum 01. September 2017 auch die Typprüfung unter Berücksichtigung der Emissionen im realen Straßenverkehr (RDE) verpflichtend. Damit erfolgt die Homologation nicht länger mittels synthetischer Prüfprozeduren unter kontrollierten Randbedingungen. Ziel dieser Maßnahme ist die Angleichung der tatsächlich anfallenden Emissionen im realen Fahrbetrieb im Vergleich zur Zertifizierung des Fahrzeugs. Hier hat sich in der Vergangenheit eine zunehmende Diskrepanz eingestellt, welche es aus Gründen der Luftreinhaltung und des Klimaschutzes zu minimieren gilt. [1][2]

Auch nach der offiziellen Einführung von RDE im Rahmen der Homologation ergeben sich nach wie vor große Herausforderungen für die effiziente und robuste Entwicklung von Antriebssträngen. Ein Hauptgrund dafür liegt in der Vielzahl veränderlicher Randbedingungen, unter denen der Antrieb gesetzeskonform funktionieren soll. Zwar schränken sowohl statische wie auch dynamische Grenzen den Variationsraum der auftretenden Prüfbedingungen ein. Dennoch sind unter den vorgegebenen Randbedingungen innerhalb der RDE-Homologation deutlich emissionskritischere Betriebsphasen zu beobachten. Mit der bevorstehenden Reduktion von Toleranzfaktoren wird das zu erreichende Emissionsziel nochmals anspruchsvoller.

Die Straße ist dabei als Entwicklungsumgebung aufgrund nicht vorhandener Reproduzierbarkeit ungeeignet. Darüber hinaus besteht das Risiko, identifizierte Probleme am fast fertigen Fahrzeug nur mit großem Aufwand beheben zu können. Gleichzeitig hat sich der Umfang von Entwicklungs- und Absicherungsmaßnahmen im Fahrzeug generell stark erhöht, was sich negativ auf Verfügbarkeit von Versuchsfahrzeugen und Kosten auswirkt [3]. Im Sinne eines effizienten Entwicklungsprozesses sollten demnach RDE relevante Problemstellungen frühestmöglich im Entwicklungsprozess adressiert und abgesichert werden. Es gilt, die reale Straßenfahrt in Prüfumgebungen hinreichend genau abzubilden, um dort den Schadstoffausstoß im realen Fahrbetrieb abbilden zu können. Dies erfordert neue methodische Ansätze, die innerhalb verschiedener Projekte am Institut für Verbrennungskraftmaschinen und Fahrzeugantriebe (VKM) der TU Darmstadt entwickelt werden.

Als Forschungsumgebung dienen für die unterschiedlichen Entwicklungsschwerpunkte Engine-in-the-Loop (EiL) Motorenprüfstände, die einen Spezialfall von Hardware-inthe-Loop Prüfständen darstellen. Diese Prüfstandsarchitektur wird langjährig am Institut VKM weiterentwickelt und stellt die Grundlage dar, um RDE am Prüfstand umzusetzen. 


\section{VKM Use-Cases und Methodikbaukasten}

Der Einfluss von RDE erstreckt sich mittlerweile auf eine zunehmende Anzahl von Entwicklungsschritten und -umgebungen, die von der Simulation bis zur Validierung reichen. Viele Anwendungsfälle erfordern dabei neue methodische Ansätze. Manche dieser Ansätze sind speziell auf die jeweilige Problemstellung zugeschnitten, andere hingegen lassen sich in Teilen oder in Gänze übergreifend nutzen. Die Entwicklungsschwerpunkte lassen sich auf folgende drei Use Cases nach [4] zurückführen:

1. Reproduktion und Analyse

2. RDE-Referenzzyklen

3. Virtuelle Integration

Dabei unterscheiden sich die Ziele der drei Use-Cases. Das Ziel für die Reproduktion und Analyse ist es, realitätsnahe sowie reproduzierbare Bedingungen herzustellen. Innerhalb der RDE-Referenzzyklen steht die hinreichend genaue Übertragung relevanter Größen an eine Prüfumgebung im Fokus der Betrachtung. Hingegen sollen im Rahmen der virtuellen Integration Grenzen und Schwachstellen bei der RDE-Bewertung des Antriebskonzepts identifiziert werden. Hierbei bedarf es insbesondere der Identifikation relevanter Testszenarien für den betrachteten Antriebstyp (sog. Most-Relevant-Test), da sich RDE-kritische Events in Abhängigkeit der gewählten Antriebstrangkonfiguration grundlegend unterscheiden [4].

Zur Adressierung der drei übergeordneten Use-Cases sind, je nach konkreter Aufgabenstellung, verschiedene Erfordernisse in unterschiedlicher Detailtiefe umzusetzen. Damit ergibt sich, dass im Allgemeinen nicht für jeden Anwendungsfall innerhalb der drei übergeordneten Use-Cases die gleichen Methoden angewandt werden können. Gleichwohl kann es vorkommen, dass Anwendungsfälle unterschiedlicher Use-Cases den selben methodischen Ansatz nutzen oder Methodiken erst in einem fortgeschrittenen Entwicklungsstadium relevant werden. In der Vergangenheit wurden verschiedene solcher Methodikbausteine am VKM entwickelt und in unterschiedlichen Kontexten eingesetzt [5][6]. Hierzu zählen unter anderem Realfahrstudien mit detaillierten Wirkkettenanalysen, Einflussanalysen der Betriebspunkthistorie sowohl bei stationärem wie auch transientem Motorbetrieb oder die gezielte Variation von veränderlichen Randbedingungen. In dieser Arbeit sollen drei aktuelle Methoden in ihrem jeweiligen Anwendungskontext vorgestellt werden. 


\section{Methodik 1 - Thermisch reale EiL-Prüfstandsumgebung}

Einen Anwendungsfall in der Motorenprüfstandsumgebung stellen thermisch reproduzierbare Komponentenuntersuchungen dar, die in einer realen Fahrzeugumgebung einzuordnen sind. So ist die Katalysatoreingangstemperatur eine wichtige Randbedingung für Untersuchungen unterschiedlicher Abgasnachbehandlungssysteme, um ein Beispiel aktueller Forschungsarbeiten zu nennen. Die Umfänge einer thermisch korrekten Abbildung motorischer Prozesse am EiL-Prüfstand sind deutlich umfangreicher, lassen sich aber in diesem Kontext exemplarisch mit der Ladeluftkühlung beschreiben. Die Forschungsarbeiten der Darstellung des dynamischen Ladeluftverhaltens werden in Kooperation mit der Firma IVD Deutschland $\mathrm{GmbH}$ durchgeführt. Anforderungen, die durch diesen konkreten Anwendungsfall charakterisiert werden, lassen sich in zwei Oberbegriffe unterteilen:

- Hohe Reproduzierbarkeit

- Thermische-Realbedingungen

Eine hohe Reproduzierbarkeit stellt eine Schnittstelle zu den bereits genannten Anwendungsfällen dar. So kann eine ausgewählte Strecke entweder real mit einem Fahrzeug gefahren oder virtuell ermittelt und ein Geschwindigkeits-Zeit (vt) Profil synthetisch erzeugt werden. Der Realversuch mit ausgewählter Messtechnik unterstützt den Prüfstandsaufbau in der Validierung und dient der methodischen Herangehensweise. Hier muss zwischen der Versuchsdurchführung und der Methodikentwicklung unterschieden werden. Ziel ist es, einen Prüfstandsmotor real, sowie das Fahrzeug in seiner simulierten Umgebung so aufzubauen, dass auch bei virtuell ermittelten Versuchen reale thermische Bedingungen erzeugt werden. Daraus resultiert im Weiteren eine Unterscheidung in die methodische Herangehensweise und die experimentelle Umsetzung.

\section{Methodische Herangehensweise}

Reale thermische Bedingungen am Motorenprüfstand stellen eine Grundvoraussetzung dar, Realfahrversuche in frühen Entwicklungsstadien am EiL-Motorenprüfstand zielgerecht durchzuführen. Am Beispiel der Katalysatoreintrittstemperatur, die als Eingangsgröße der Abgasnachbehandlungsentwicklung dient, wird die Methodik eines thermisch realen EiL-Prüfstandaufbaus deutlich. Abbildung 1 stellt einen schematischen Prüfstandsaufbau mit dem Minimum an Temperaturmessstellen im Frischluftund im Abgaspfad dar. Die Ansaugtemperatur, die nach der Verdichtung über den Ladeluftkühler herunter gekühlt wird, wird anschließend mit den zurückgeführten Abgasen dem Brennraum zugeführt. 


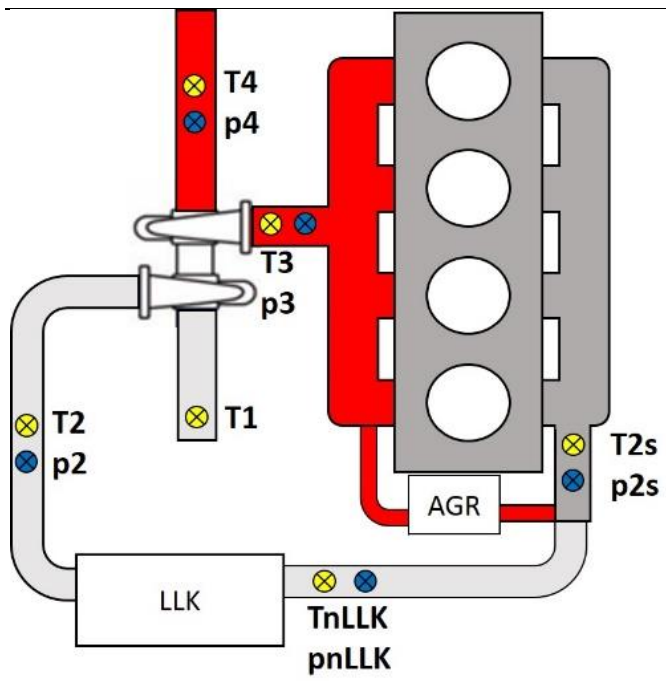

Abbildung 1: Schematischer Luftpfad mit Sensoren am EiL-Prüfstand

Der typische Wasser-Luft-Prüfstandsladeluftkühler, wie er häufig auf Prüffeldern Anwendung findet, ist aufgrund seiner hohen thermischen Masse schlecht regelbar und zeigt deutliche Abweichungen zu Realfahrzeugmessungen. Abbildung 2 zeigt eine reale Fahrzeugmessung im Vergleich zu einer Prüfstandsmessung. Beide Messungen finden bei $23^{\circ} \mathrm{C}$ statt und weisen unterschiedliche Geschwindigkeitsprofile auf, die jedoch auf der gleichen Strecke verlaufen und vergleichbar sind. Die Ansaugtemperatur ist ein ausschlaggebender Faktor zum einen für die sich einstellende Verbrennung, zum anderen aber bereits zuvor, je nach verbauter Sensorik, für die vom Motorsteuergerät (ECU) ausgewählten Kennfelder, in denen der Motor betrieben wird. Bei zu hohen Abweichungen der Solltemperaturen kann es sein, dass der Motor in von der Straßenfahrt abweichenden Kennfeldern betrieben wird, wodurch ein Vergleich unmöglich wird.

Das Ziel ist somit klar definiert: Reale Wärmeströme und Temperaturen auch am ,stillstehend verbauten“ EiL-Prüfstandsmotor zu erzeugen. Die Ladeluftkühlung in modernen Fahrzeugen ist entweder als direkter Luft-Luft-Wärmetauscher oder als indirekter Luft-Wasser-Wärmetauscher, der über einen weiteren Wasserkreislauf fahrtwindgekühlt wird, ausgeführt. Darüber hinaus ist unter anderem auch die gezielte Bauteilanströmung zu berücksichtigen, besonders im Bereich höherer, simulierter Fahrzeuggeschwindigkeiten [7]. 


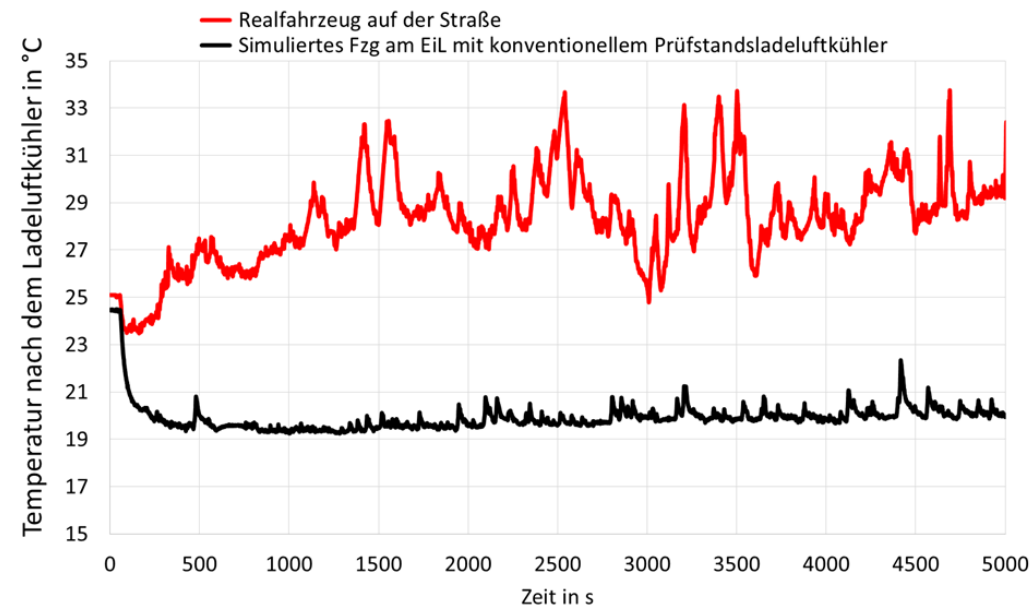

Abbildung 2: Temperatur nach dem Ladeluftkühler real und EiL mit unterschiedlichen Ladeluftkühlersystemen

Das niedrige Temperaturniveau der Ladelufttemperatur nach dem Ladeluftkühler am Motorenprüfstand bei einer Luft-Wasser-Ladeluftkühlung ist auf die Hochlastfähigkeit des Kühlers zurückzuführen. Die Kühlung wird auf eine Volllast-Ladelufttemperatur eingestellt und verhält sich dementsprechend kalt in Niedrigtemperatur-Kennfeldbereichen, wie sie bei urbanen RDE-Messungen abgerufen werden. Eine Regelung des Kühlwassermassenstroms hat sich aufgrund der hohen thermischen Masse der Ladeluftkühlereinheit als nicht nützlich erwiesen. Gleiches gilt für Fahrzeug-Luft-LuftLadeluftkühler, die in einem Wasserbad betrieben werden. Außerdem ist bei dieser Vorgehensweise die Fehleranfälligkeit, die zu einem Wasserschlag führen könnte, zu erwähnen.

Die Nutzung eines Realfahrzeug-Ladeluftkühlers hat den Vorteil originaler FahrzeugLadeluftwege. Der Fahrtwind lässt sich mit einem Gebläse einregeln. Hohe Lastanforderungen sind jedoch nicht mit einer reinen Gebläsekühlung darzustellen, da diese die Wärmeabfuhr nicht leisten kann. In Hochlastbetriebspunkten, wie sie real bei Beschleunigungen, wie Autobahnauffahrten, vorkommen, oder bei Stationärmessungen am Motorenprüfstand, lässt sich die Gebläsekühlung durch eine Wasseraufspritzung ergänzen. Die Verdampfungsenthalpie des Wassers ergänzt die erzwungene Konvektion durch das Gebläse. Versuche haben hier eine hohe Dynamik ergeben, wodurch sich das System auch bei der Abbildung realer Fahrszenarien anbietet. Final lässt sich festhalten, 
dass diese Systematik eine Optimierung für eine reproduzierbare Prüfstandsumgebung darstellt, wodurch sich Bauteil- oder Applikationsvariationen unter realen Bedingungen am EiL-Prüfstand vergleichen und bewerten lassen.

\section{Experimentelle Umsetzung}

Die konstruktive und simulative Umsetzung thermisch realer Umgebungsbedingungen unter dem Aspekt der hohen Reproduzierbarkeit am Engine-in-the-Loop Motorenprüfstand gibt die Möglichkeit der Bauteilvariation unter realen Bedingungen. Dabei steht ein exakter Übertrag einer realen Messfahrt eher im Hintergrund. Das konkrete Ziel stellt eine real repräsentative Messumgebung dar. Realfahrzeugmessungen der gleichen Strecke bei gleichen Umgebungstemperaturen mit unterschiedlichen Geschwindigkeits-Zeit-Verläufen haben gezeigt, dass sich Ladelufttemperaturen in einem engen Streuband befinden. Dieses Streuband gilt es mit der Prüfstandsladeluftkühlung darzustellen. Somit ergibt sich die Möglichkeit, synthetische Geschwindigkeits-Zeit-Profile darzustellen, ohne diese je real gefahren zu sein und Ergebnisse in diesem Streuband zu erlangen. Einen wichtigen Fakt stellt die Tatsache dar, dass eine Realfahrt auf der Straße niemals reproduzierbar ist. Diesen Aspekt kann die Prüfstandsmessung jedoch leisten. Dadurch sind geringste Änderungen der Applikation in thermischen- oder emissionsmesstechnischen Konsequenzen darzustellen.

\section{Methodik 2 - Darstellung realer Streckenprofile mit individuellen Fahrprofilen}

Einen weiteren Anwendungsfall stellt das realitätsnahe Abbilden von RDE-Tests in der Prüfstandsumgebung dar. Um eine Straßenfahrt in eine Prüfumgebung zu übertragen, muss hierbei ein Datensatz mit der entsprechenden Streckeninformation bereitgestellt werden. Existieren bestehende Messdaten für eine spezifische Strecke (z.B. PEMSMessung), so lassen sich diese in eine derartige Sollwertvorgabe überführen. Für beliebige Strecken ist dieser Ansatz jedoch aufgrund der benötigten Messdaten und des damit verbundenen Aufwandes ungeeignet und schränkt somit den Untersuchungshorizont stark ein. Aufgrund der Komplexität und Vielschichtigkeit einer realen Straßenumgebung (Verkehrszeichen, Lichtsignalanlagen, Kreuzungen, Vorfahrtsregelungen, variierende Steigungen und Kurvenradien, etc.) bietet es sich an, diese Informationen aus bestehenden Kartendaten zu extrahieren, anstatt sie aufwendig synthetisch zusammenzustellen.

Bei beiden Varianten ist dabei einer adäquaten Abbildung der Höheninformation eine große Bedeutung zuzuordnen, da sich hieraus über die Fahrwiderstände des Fahrzeugs 
das Lastprofil des Prüflings ergibt. Gelingt die Aufprägung des Lastprofils nur unzureichend, ergeben sich entsprechend andere Betriebspunkte für den Antrieb und die Emissionsergebnisse lassen sich nicht realitätsnah darstellen.

Für die Erstellung der Steigungsprofile werden derzeit am VKM unterschiedliche Quellen an Höheninformationen genutzt, die mit unterschiedlichen Vor- und Nachteilen behaftet sind. Neben den messtechnisch zu erfassenden Daten aus barometrischer und satellitengestützter Höhenbestimmung, kommen die kartenbasierten Höheninformationen der Shuttle Radar Topography Mission (SRTM) und des hochauflösenden Digitalen Geländemodells (DGM) des Airborne Laserscannings der Hessischen Verwaltung für Bodenmanagement und Geoinformation zum Einsatz. Onlinekarten wie GoogleMaps, Open-Street-Maps (OSM) oder Bing nutzen in der Regel SRTM-Daten [8]. Diese werden teilweise mit weiteren Daten wie GPS-Informationen von Handynutzern fusioniert. Die Reproduktionsgüte variiert je nach gewählter Datenquelle. Als Merkmal für die erzielbare Güte wird in Abbildung 3 die berechnete Leistungsanforderung im Vergleich zur aufgezeichneten Leistungsanforderung bei gleichem Geschwindigkeitsprofil dargestellt.

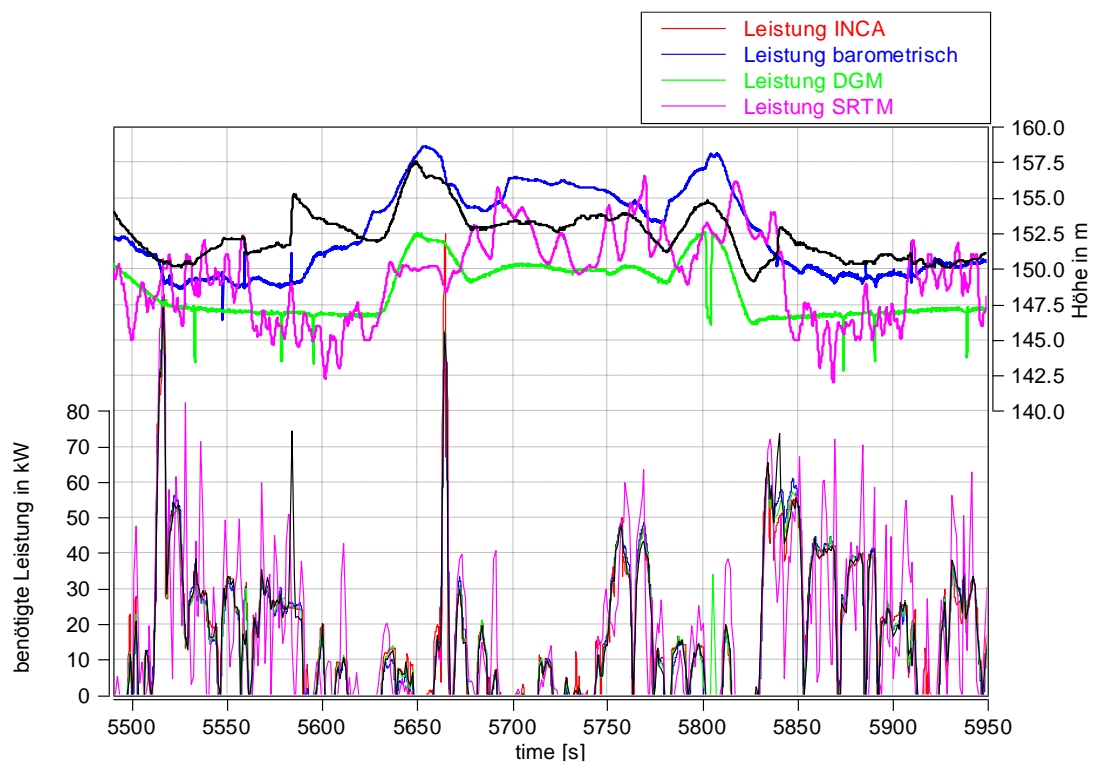

Abbildung 3: Leistungsanforderung in Anhängigkeit des gewählten Höhenprofils 
Bei genauerer Analyse wird ersichtlich, dass mit Hilfe der DGM Daten die beste Übereinstimmung mit der im Fahrversuch aufgezeichneten Leistungsanforderung erreicht werden kann. Durch geeignete Bearbeitung der barometrischen Höhenmessung kann ebenfalls eine gute Übereinstimmung mit den aufgezeichneten Werten erreicht werden. Mit Hilfe der GPS Höhe kann in weiten Bereichen ebenfalls eine gute Korrelation erlangt werden, die nur an einigen Stellen zu Problemen führt. Bezüglich der SRTM Daten sind bereits im Höhenprofil deutliche Abweichungen der Steigung zu erkennen, was sich auch in der berechneten Leistungsanforderung bemerkbar macht.

Soll ein Prüflauf auf einer Strecke erstellt werden, auf der bisher keine Testfahrten stattgefunden haben, entfallen die Möglichkeiten der barometrischen und der satellitengestützten Höhenbestimmung. Tabelle 1 fasst die Vor- und Nachteile der oben beschriebenen Höhenquellen zusammen.

Tabelle 1: Bewertung der genutzten Höhendaten

\begin{tabular}{|l|c|c|c|l|}
\hline Quelle & Verfügbarkeit & Prädiktion & Genauigkeit & Einschränkung \\
\hline GPS & + & -- & - & $\begin{array}{l}\text { Tunnel/Brücken, GPS-Ab- } \\
\text { schattung }\end{array}$ \\
\hline Druck & o & -- & o & $\begin{array}{l}\text { Quereinflussanalyse Ge- } \\
\text { schwindigkeit notwendig }\end{array}$ \\
\hline SRTM & ++ & ++ & -- & Tunnel/Brücken, Auflösung \\
\hline DGM & - & ++ & ++ & Tunnel/Brücken \\
\hline
\end{tabular}

Zur Generierung einer Sollwertvorgabe für eine Prüfumgebung aus digitalen Kartendaten wurde gemeinsam mit der Firma AVL List GmbH eine Methode entwickelt, die Bauer [6] vorgestellt hat. Ein Programm, das diese Methode nutzt ist bereits umgesetzt und befindet sich in der Erprobungsphase. Hierbei wird in Onlinekartendaten (hier OSM) die gewünschte Route definiert. Anschließend werden die Geodaten und Karteninformationen der gesamten Strecke extrahiert. Es ergibt sich ein Koordinatentrack, der außerdem die Geschwindigkeitsbegrenzung zu jedem Wegpunkt enthält. Auf Basis festzulegender Fahrer- und Fahrzeugparameter werden anhand der Fahrwiderstandsgleichung und fahrerabhängiger Kurvengeschwindigkeiten die tatsächlich realisierbaren Geschwindigkeitsverläufe berechnet. Daneben kann das unterschiedliche menschliche Fahrverhalten über variable Beschleunigungs- und Verzögerungsraten berücksichtigt werden. Zusammengefasst wird auf diese Weise ein Streckenprofil inklusive einer Geschwindigkeitsvorgabe generiert. Im Vergleich zum Streuband vieler Fahrzeugmessungen derselben Strecke ergibt sich ein hoher Übereinstimmungsgrad. 
Methodikbaukasten zur effizienten, zielgerichteten RDE Entwicklung

Potentiale und Perspektiven

Maßgebliche Unterschiede entstehen primär durch die Einflüsse des Verkehrsaufkommens, von Kreuzungssituationen und dem fluktuierenden Geschwindigkeitsregelverhalten des menschlichen Fahrers bei annähernd konstanter Geschwindigkeit.

Die Adressierung des erstgenannten Aspekts gestaltet sich dahingehend schwierig, als dass keine Messdaten unter Ausschluss jeglichen Einflusses des umgebenden Verkehrs bei Straßenmessungen vorliegen. Ebenfalls ist eine nachträgliche Separation zwischen fahrerinduzierten und verkehrsbedingten Abweichungen vom Durchschnittsprofil nur schwer zu realisieren. Damit fehlt insgesamt eine Basis für die valide Modellierung des Verkehrseinflusses. Zur Steigerung der Abbildungsgüte werden die Nachbildung von Kreuzungs- und Konstantfahrsituationen tiefer analysiert.

Die nativen Kartendaten aus den verwendeten Quellen enthalten keine direkten nutzbaren Markierungen für Kreuzungspunkte. Zwar existieren Marker für Ampeln, welche auf eine Kreuzungssituation hinweisen. Jedoch reichen die verfügbaren Metadaten des Kartenmaterials nicht für klassische rechts-vor-links Situationen aus, in denen der menschliche Fahrer die Geschwindigkeit bis hin zum vollständigen Stoppen reduzieren kann. Kreuzungspunkte lassen sich allerdings über die Prüfung des folgenden Kriteriums identifizieren: Kartenpunkte, die zu einer Straße gehören, sind in Kartendaten entsprechend mit dieser Information versehen. Gehört ein Kartenpunkt zu mehreren Straßen, so handelt es sich in der Regel um eine Kreuzung. Für die Routenplanung ergeben sich an einer gewöhnlichen 3-Wege-Kreuzung im Allgemeinen drei Möglichkeiten für die weitere Fahrtroute: Linksabbiegen, Rechtsabbiegen oder die Geradeausfahrt. Die Kreuzungslogik unterscheidet erst, ob die Geschwindigkeit nach einer Kreuzung größer ist als vor der Kreuzung. Falls dies der Fall ist, wird beim Abbiegen angenommen, dass man auf eine Straße höherer Priorität auffährt und somit an der Kreuzung halten muss. In den meisten Ländern mit Rechtsverkehr hat in Tempo 30 Bereichen derjenige Vorfahrt, der von rechts kommt. Es wird die Annahme getroffen, dass somit Fahrzeuge vor dem Abbiegen nach links zu einem Stopp kommen, während Fahrzeuge in Geradeausfahrt ihre Geschwindigkeit verringern, um gegebenenfalls schnell ihrer Wartepflicht nachzukommen. Bei einem Abbiegevorgang nach rechts wird einzig die Geschwindigkeit entsprechend der bereits hinterlegten Geschwindigkeitsreduktion für Kurvendurchfahrten reduziert, ein zusätzlicher Eingriff erfolgt nicht. Unterschiedliches Fahrerverhalten an Kreuzungen wird dabei in Abhängigkeit des gewählten Fahrertyps berücksichtigt, wodurch stärkere oder schwächere Beschleunigungen bzw. Verzögerungen induziert werden. In Abbildung 4 ist ein Ausschnitt aus dem innerstädtischen Bereich dargestellt. Hier wird die Simulation mit und ohne Kreuzungslogik mit Messungen aus dem gleichen Bereich gegenübergestellt. 
Methodikbaukasten zur effizienten, zielgerichteten RDE Entwicklung

Potentiale und Perspektiven

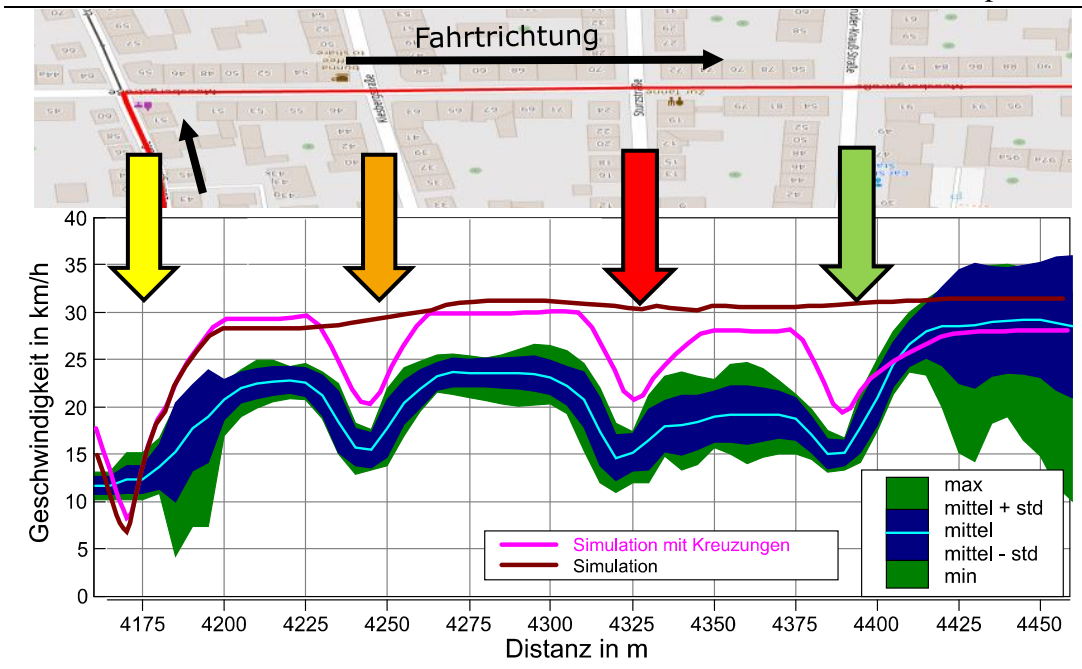

Abbildung 4: Auswirkung der Kreuzungslogik

Aus den Ergebnissen ist zu erkennen, dass mit der neu eingeführten Logik eine deutlich realitätsnähere Geschwindigkeitsführung im urbanen Bereich realisiert werden kann. Auch wenn die durchschnittlichen Geschwindigkeiten der Simulation noch über den gemessenen Fahrten liegen, wird die Dynamik in diesem Bereich mit Berücksichtigung der Kreuzungen besser abgebildet.

Fahrabschnitte, in denen ein menschlicher Fahrer auf eine konstante Geschwindigkeit regelt, resultieren in der Regel in einer leicht fluktuierenden, tatsächlich erzielten Geschwindigkeit des Fahrzeugs. Ursächlich hierfür können beispielsweise geringfügige Änderungen in der Steigung, dem Fahrtwind oder Verkehrseinflüsse sein. Weiterhin nimmt der Fahrer Abweichungen nicht unmittelbar war und toleriert Regelabweichungen in gewissen Grenzen. Letztendlich führen diese Einflüsse zu einem veränderten Eingriff der Stellgröße des Steuergerätes, das Gaspedal und damit der anliegenden Motorlast. Um dieses fahrerinduzierte Verhalten besser abzubilden, wird der Regler wie folgt angepasst.

Mittels einer Leistungsführung, die das menschliche, zeitbasierte Regelverhalten als Leistungsregler abbildet, wird eine realistische Varianz in der Wunschgeschwindigkeit erzeugt. Dies führt auch zu einem ausgewogeneren Lastkollektiv des Motors, der infolgedessen einen großen Teil des Teillastbereiches abdeckt und weniger stark im Wechsel 
zwischen Niedrig- und Hochlast betrieben wird. Diesem Ansatz liegt die Annahme zugrunde, dass Streuungen der Geschwindigkeiten auf Bereichen mit konstanter Geschwindigkeitsvorgabe hauptsächlich durch die Straßensteigung bewirkt werden. Dies geschieht durch eine feste Leistungsvorgabe an prägnanten Stellen, damit die Fahrzeuggeschwindigkeit soweit wie möglich in der inneren Toleranz gehalten wird. Beim Eintritt in das Toleranzband wird der Leistungswunsch auf die bei der aktuellen Zielgeschwindigkeit bei aktueller Steigung berechnete Leistung gesetzt und so lange konstant gehalten, bis sich die Geschwindigkeit, beeinflusst durch die Steigung, dem Toleranzband nähert. Wird eine vorgegebene Grenze überschritten, wird eine neue Leistungsvorgabe ausgewählt. Dadurch wird die resultierende Fahrzeuggeschwindigkeit steigungsabhängig, da große Steigungsunterschiede schnell zu einer Leistungsanpassung führen.

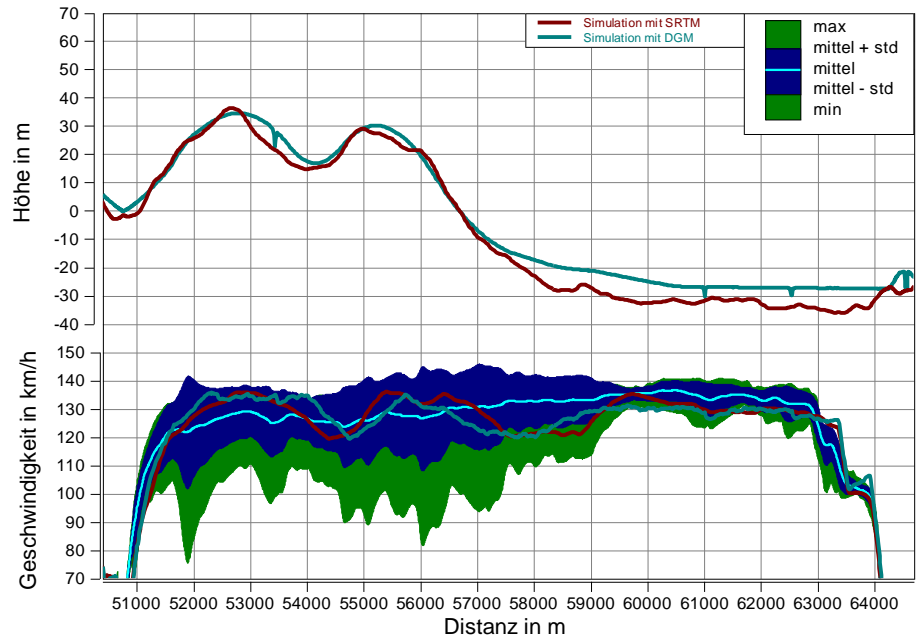

Abbildung 5: Reglerverhalten bei konstanter Geschwindigkeitsvorgabe

Auf dem in Abbildung 5 abgebildeten Autobahnabschnitt ist eine konstante Geschwindigkeit vorgegeben. In diesem Bereich wird die Leistungsführung aktiv. Um einen verbesserten Überblick über die Verteilung der Messfahrten um den Mittelwert zu bekommen, wird zusätzlich zu dem Band von Minimum und Maximum die empirische Standardabweichung zum Mittelwert (blaues Messband) eingeführt. Aus den Messwerten ist eine Tendenz erkennbar, nach der sich bei geringen Steigungen eine kleinere Streuung um den Mittelwert einstellt. Abhängig vom Steigungsausmaß und der Ände- 
rungen im Steigungsverlauf ergibt sich eine größere Abweichung. Ein ähnliches Verhalten kann an der simulierten Geschwindigkeit aufgrund der Leistungsführung beobachtet werden, denn diese passt die Leistung an den kritischen Stellen mit hohen Steigungsänderungen öfter an, als bei Streckenteilen mit ähnlicher Steigung aber geringen Änderungsraten.

Insgesamt ist im Vergleich der Vorgängerversion mit dem aktuellen Stand der Simulation (siehe Abbildung 6) zu erkennen, dass die simulierte Geschwindigkeit weiterhin gut in dem von Messungen erstellten Messbereich liegt. Durch die beiden oben beschriebenen Neuerungen Kreuzungslogik und Leistungsführung konnten zum einen die Dynamik im städtischen Bereich besser an die durch Messdaten generierte Datenbasis angepasst werden. Auf der anderen Seite konnte die in der Vorgängerversion noch deutlich als synthetisch zu erkennende Geschwindigkeitsführung bei konstanter Vorgabe in eine deutlich realistischere Vorgabegeschwindigkeit überführt werden.

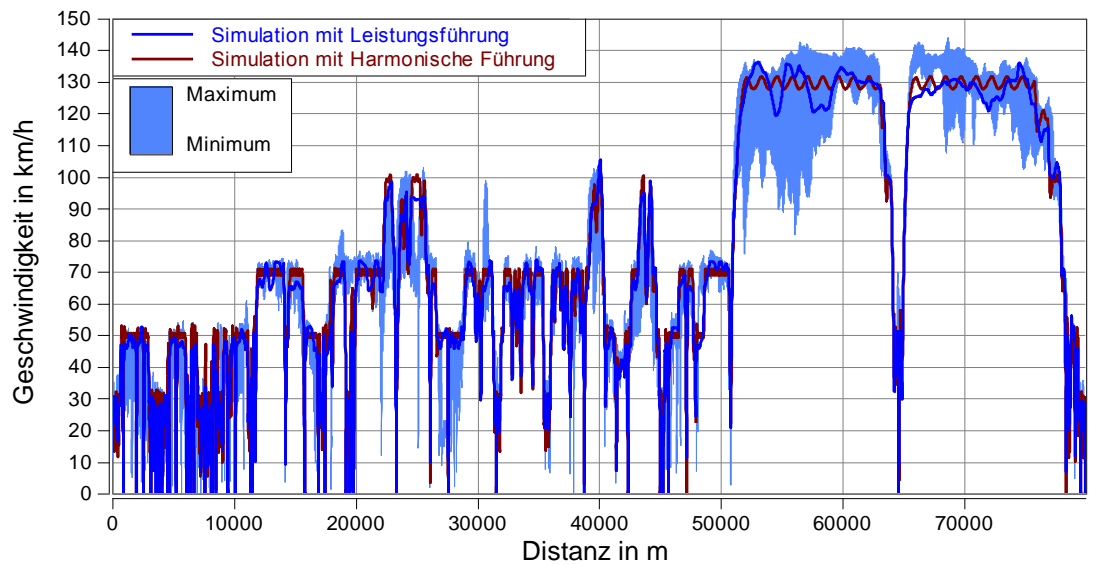

Abbildung 6: Vergleich Geschwindigkeitsprofil neue Version mit Vorgängerversion

Sofern die Generierung einer Strecke gewünscht ist, die innerhalb der RDE Prozedur vollständige Gültigkeit besitzt, kann dieses Kriterium innerhalb eines weiteren Moduls automatisiert überprüft werden. Zu diesem Zweck ist eine Fahrzeugcharakteristische $\mathrm{CO}_{2}$-Kurve für das parametrierte Fahrzeug hinterlegt, mit der ein $\mathrm{CO}_{2}$ Erwartungswert für eine Fahrt auf dieser Strecke berechnet wird. Auf Basis einer simulierten Fahrt liefert das RDE Post-Processing automatisiert ein Konformitätsergebnis. 


\section{Methodik 3 - Antriebsstrang- und emissionsspezifische Worst-} Case Betrachtung innerhalb einer RDE-validen Testprozedur

Die vorgestellte Methodik, welche in Zusammenarbeit mit der Honda R\&D Europe (Deutschland) $\mathrm{GmbH}$ entstanden ist, findet in zweierlei Hinsicht Anwendung: Zum einen ist es über das Verfahren der antriebsstrang- und emissionsspezifischen Worst-Case Betrachtung möglich, bereits frühzeitig eine hohe Sicherheit über das Bestehen des gesetzten Emissionsziels während der Zertifizierung mit den ausgewählten Komponenten zu erlangen. Individuelle Fahrsituationen, die in Bezug auf den gewählten Antriebsstrang und den untersuchten Schadstoff zu erhöhten Emissionsereignissen führen, werden gezielt innerhalb einer RDE-validen Testprozedur angeregt und bewertet. Zum anderen erlaubt die Methodik die Identifikation kritischer Eckpunkte in der Applikation in einem frühen Entwicklungsstadium des Antriebsstrangs, denen in der Folge durch entsprechende Maßnahmen effizient begegnet werden können.

Der Ansatz, maßgeschneiderte Tests für die jeweiligen Antriebstechnologien zu verwenden, ist durch verschiedene Entstehungsmechanismen der Schadstoffbildung und applikationsabhängige Beeinflussung dieser begründet. Dabei spielen insbesondere das gewählte Brennverfahren, die Gemischzusammensetzung, innermotorische Reduktionsmaßnahmen als auch Arbeitsbereiche der verwendeten Abgasreinigungsstrategie eine tragende Rolle. Vor allem Trade-Off-Effekte können nicht ausreichend mit standardisierten Tests, die eine Sammlung unspezifischer Fahrmanöver enthalten, angesprochen werden. Die Notwendigkeit einer gezielten Auswahl von Fahrsituationen als Bewertungsgrundlage zur Sicherung des Bestehens der RDE-Prozedur wird in Abbildung 7 verdeutlicht.

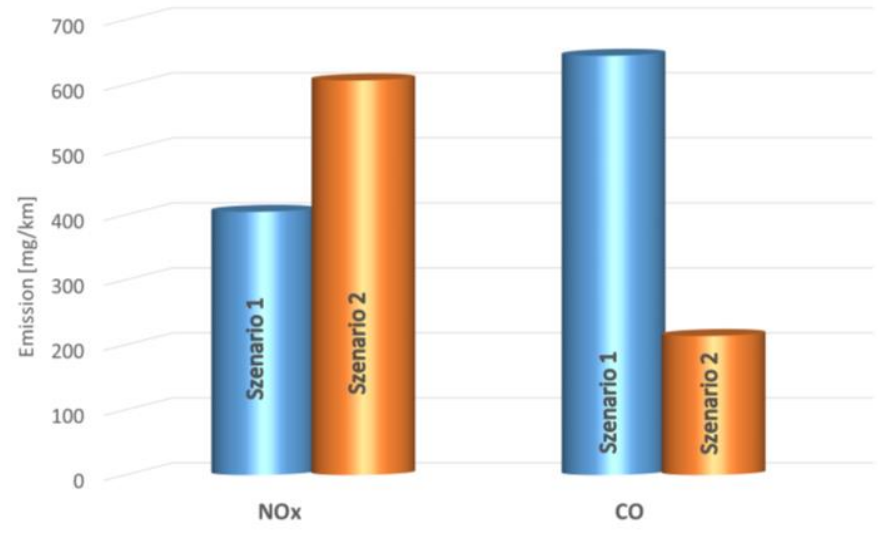

Abbildung 7: Emissionsergebnisse von zwei Schadstoffen bei unterschiedlichen Strecken [9] 
Die dargestellten Emissionsergebnisse eines Euro-5-Dieselmotors im C-Segment beziehen sich auf zwei unterschiedliche RDE-Strecken. Beide Strecken genügen den Rahmenbedingungen der Gesetzgebung und sind nach aktuellem Stand als gültig zu bewerten. Szenario 1 ist hauptsächlich durch einen ebenen Streckenverlauf und eine moderate Fahrweise gekennzeichnet. Szenario 2 zeichnet sich durch viele Steigungen, regen Verkehr und eine wenig vorausschauende Fahrweise aus. Auffallend ist, dass bei Szenario 2 im Vergleich zu Szenario 1 die ausgestoßenen NOx-Emissionen infolge transienter und hochlastiger Anforderungen deutlich zunimmt, der CO-Ausstoß hingegen bei einer in Bezug auf energetische Gesichtspunkte anspruchsvolleren Fahrt drastisch abnimmt. Daraus lässt sich schlussfolgern, dass ein Test, welcher bezüglich des $\mathrm{NO}_{\mathrm{x}}$-Austoßes als anspruchsvoll und kritisch zu bewerten ist nicht zwangsläufig auch herausfordernd für die Betrachtung anderer Schadstoffe ist. Eine Festlegung auf ein Testszenario, welches besonders dynamisch und anspruchsvoll hinsichtlich der Stickoxide ist, ist demnach nicht zielführend für die Gesamtbewertung des Antriebsstrangs und birgt die Gefahr des Unterlassens wichtiger Tests zur Überprüfung anderer Aspekte des Antriebsstrangs. Gleiches gilt für die Verwendung eines bestimmten Tests für unterschiedlichste Antriebskonzepte.

Ein weiterer wichtiger Aspekt der vorgestellten Methodik ist die Berücksichtigung und gezielte Platzierung sogenannter Historieneffekte. Darunter sind Abfolgen verschiedener Fahrmanöver zu verstehen, die als Gesamtereignis zu einer Emissionserhöhung führen. Beispielhaft ist in Abbildung 8 das Partikelemissionsergebnis für den immer gleichen Beschleunigungsvorgang bei Variierung des Steigungsverlaufs der vorangegangenen Konstantfahrt gezeigt.

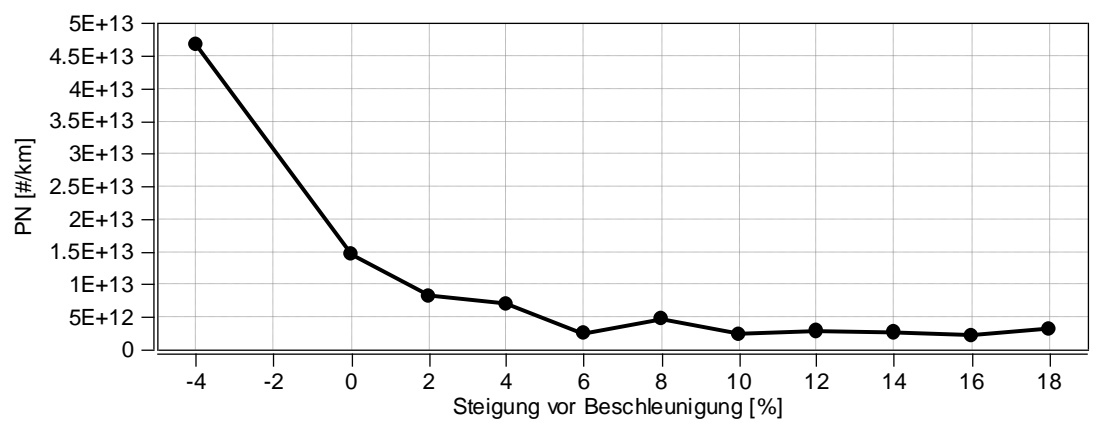

Abbildung 8: PN-Ergebnisse bei Variation des Ausgangszustandes vor einer Beschleunigung 
Das Ergebnis zeigt, dass vor allem bei vorangegangenen Bergabfahrten mit entsprechender Auskühlung des Brennraums trotz des anschließenden identischen Fahrmanövers erhöhte Partikelemissionen beim verwendeten Untersuchungsmotor zu verzeichnen sind und eine Betrachtung der Abfolge verschiedener Fahrmanöver unabdingbar ist. Das Beispiel demonstriert, dass mithilfe der Methodik und des gezielten Anregens von Emissionsereignissen des untersuchten Konzepts, die Gefahr des Auftretens unvorhergesehener Ereignisse, die in standardisierten Tests unter Umständen aufgrund der Fülle möglicher Fahrsituationen keine Berücksichtigung finden, minimiert werden kann. Der Prozess der Methodik, konzeptindividuell ein Most Relevant Testszenario zu erschaffen und zu bewerten, ist in Abbildung 9 dargestellt.

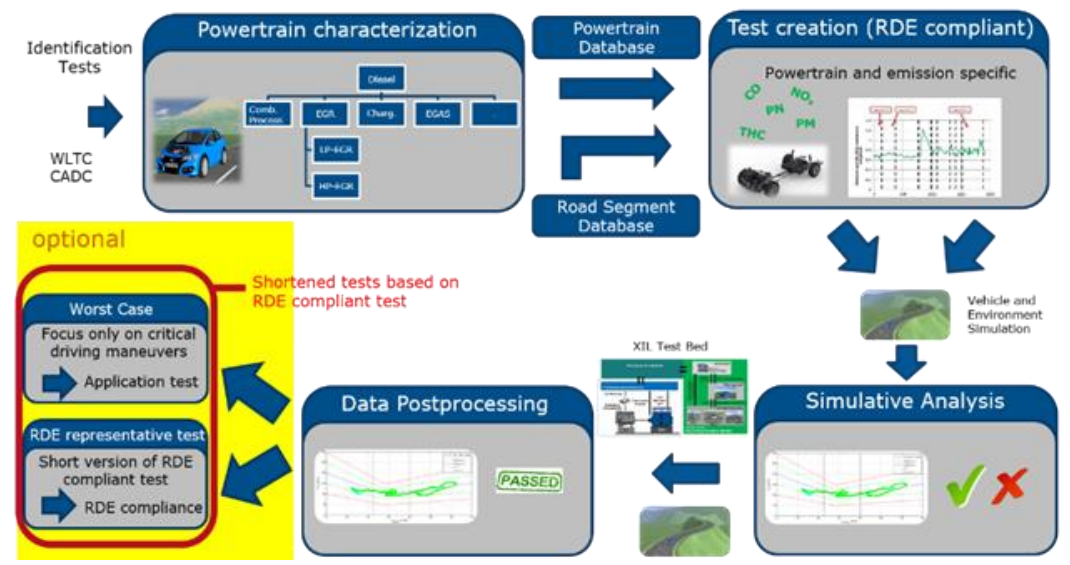

Abbildung 9: Most Relevant Test Methodik zur Generierung von antriebsstrang- und emissionsspezifischen Testszenarien

Der Prozess ist in fünf Module gegliedert, welche durch die blaugrauen Blöcke illustriert sind. Damit wird eine Funktionstrennung verschiedener Aufgaben der Methodik erreicht, die einen einfachen Austausch einzelner Module durch etwaige Änderungen (z.B. Postprocessing Verfahren) erlaubt. Beginnend wird das Modul „Powertrain Characterization" beschrieben, welches Informationen zu den Komponenten des verwendeten Antriebsstrangs und seines energetischen Verhaltens aufweist. Mithilfe von Informationen, die je nach Verfügbarkeit aus Prüfstandsmessungen oder aber aus Simulationen stammen können, kann das grundsätzliche Emissionsverhalten in der Powertrain Database beschrieben werden. Diese wird zusammen mit der Road Segment Database, welche verschiedenste Fahrmanöver enthält, an das nächste Modul, die „Test Scenario Creation“ übergeben. Dieses Modul stellt das Kernmodul des Prozesses dar 
und erzeugt automatisiert, basierend auf den Eingängen der Datenbänke und des Powertrain Configuration Moduls, einen antriebsstrangspezifischen emissionskritischen Test innerhalb der RDE-Rahmen- und Gültigkeitsbedingungen. Als Ausgabe erfolgt hier eine Strecken-, Umwelt- und Fahrerbeschreibung, welche an eine entsprechende 3D-Umwelt- und Gesamtfahrzeugsimulation übergeben werden kann. Am VKM wird dazu AVL InMotion powered by IPG CarMaker verwendet. Das erstellte Testszenario, welches direkt mit dieser Software auf XiL Prüfständen verwendbar ist, kann zunächst im Modul „Simulative Analysis“ rein simulativ im Hinblick auf die Eignung zur Prüfstandsnutzung bewertet werden. Wird das Szenario als geeignet eingestuft, kann dieses am XIL-Prüfstand ausgeführt und reale Emissionsergebnisse am Motorenprüfstand gemessen werden. Die Ergebnisse des Tests können im Modul „Data Postprocessing“ wahlweise entsprechend der aktuell geltenden Regularien oder auch als Rohergebnisse bewertet und untersucht werden. Im Anschluss daran bieten die optionalen Module „Worst Case Test“ bzw. „Shortened RDE representative Test“ die Möglichkeit, aus dem emissionskritischen RDE-validen Test der „Test Scenario Creation“ einen verkürzten Test zur effizienten Prüfstandsnutzung im Entwicklungsumfeld im Hinblick auf zwei verschiedene Zielsetzungen zu erstellen: Der „Worst Case Test“ identifiziert dabei die kritischsten Manöver und rafft diese zur Nutzung als Applikationstest in einem verkürzten Test zusammen. Das „Shortened RDE representative Modul“ hingegen erzeugt einen verkürzten Test mit einem vergleichbaren Emissionslevel in Bezug auf das Ergebnis der „Test Scenario Creation“ und stellt damit eine Auswahl RDE repräsentativer und emissionskritischer Fahrsituationen zusammen, die als Bewertungsgrundlage hinsichtlich der Gesetzgebung verwendet werden können. Für weiterführende Informationen sei auf [9] verwiesen.

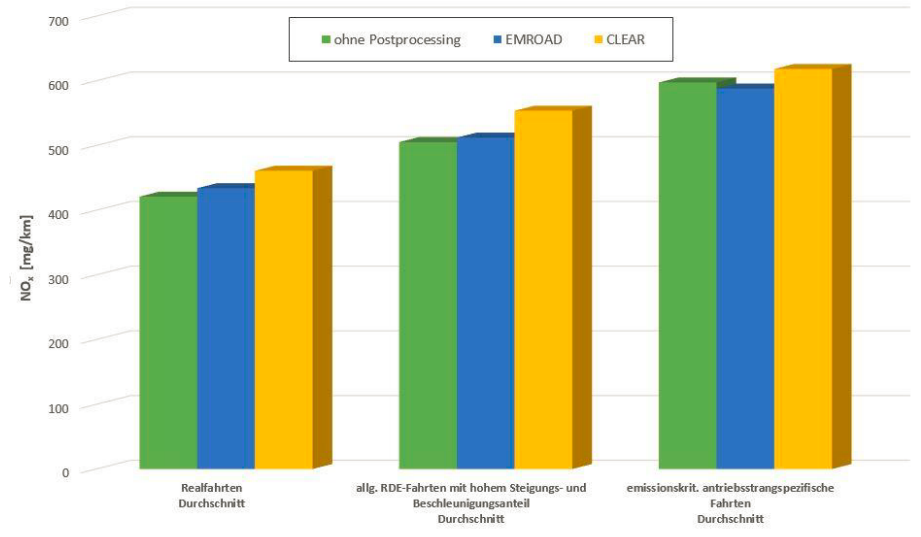

Abbildung 10: $\mathrm{NO}_{\mathrm{x}}$-Ergebnisse in Abhängigkeit von Streckentyp \& Auswertungsverfahren [9] 
In Abbildung 10 sind Ergebnisse des Moduls „Test Scenario Creation“ und der anschließenden Datenauswertung des angewandten Prozesses gezeigt. Dabei ist ein Vergleich von Realfahrten mit als im allgemeinen Sinne dynamischen RDE-Szenarien und den emissions- und antriebstrangspezifischen Szenarien zu sehen.

Vor allem bei den antriebsstrangspezifischen $\mathrm{NO}_{\mathrm{x}}$-emissionskritischen Fahrten ist eine deutliche Steigerung der $\mathrm{NO}_{\mathrm{x}}$-Emissionen im Vergleich zu beliebigen Streckenprofilen zu verzeichnen. Auch im Vergleich zu RDE-Fahrten, die nach allgemeinen dynamischen Kriterien erstellt wurden, ist eine Zunahme des Stickoxidausstoßes zu erkennen. Die nicht spezifischen Tests decken somit einige $\mathrm{NO}_{\mathrm{x}}$-kritische Fahrsituationen nicht oder nicht ausreichend ab, was den Vorteil eines antriebsstrangspezifischen Testszenarios ersichtlich macht.

In Abbildung 11 sind Ergebnisse für einen aktuellen Euro-6-Ottomotor in verschiedenen Szenarien dargestellt, die ebenfalls den Nutzen einer maßgeschneiderten Testgrundlage hervorheben. Am Beispiel CO ist festzuhalten, dass je nach Test ein Bestehen oder Nichtbestehen der aktuellen Emissionsziele erzielt werden kann und bei Verwendung einer entsprechenden Bewertungsgrundlage diese Fahrsituationen entsprechend früh entdeckt werden können.
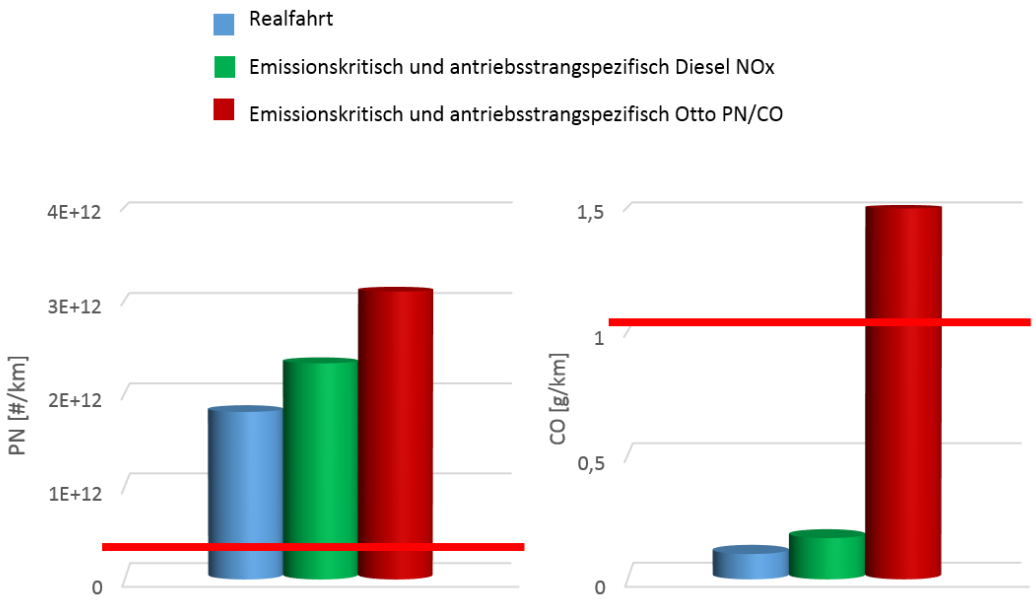

Abbildung 11: Vergleich PN und CO Ergebnisse in verschiedenen Tests 


\section{Zusammenfassung und Ausblick}

Am VKM wird fortwährend zu aufgabenspezifischen Methoden zur phasenoptimalen RDE-Entwicklung geforscht, von denen beispielhaft drei vorgestellt werden: Soll die ECU die gleichen Reaktionen wie im realen Fahrbetrieb zeigen, so ist die Methodik zur thermisch realen EiL-Prüfstandsumgebung eine wichtige Grundlage. Zur Generierung von Prüfszenarien auf Grundlage realer oder virtueller Testfahrten eignet sich die Methode zur Darstellung realer Streckenprofile mit individuellen Fahrprofilen. Die Methodik zur antriebsstrang- und emissionsspezifischen Worst-Case Betrachtung liefert RDE-konforme Tests zu Bewertungszwecken, sowie emissionskritische Events zur applikativen Optimierung.

Die Herausforderungen innerhalb der RDE-Entwicklung und der phasenoptimale Einsatz adäquater methodischer Ansätze werden teilweise als komplex und unübersichtlich empfunden. Dies liegt vor allem an der der Fülle an Variablen und Wechselwirkungen, die es je nach Untersuchungsumfang unterschiedlich zu berücksichtigen gilt. Je nach Anwendungsfall ist zu definieren, welche Vereinfachung in welchem Prozessschritt zulässig ist. Aus diesem Grund werden durch die methodischen Ansätze am VKM RDErelevante Einflüsse einzeln unter Berücksichtigung der Wechselwirkungen adressiert. Erfüllt ein methodischer Ansatz sein Ziel oder ist der Vergleich zu anderen RDE-Einflüssen und deren methodischen Ansätzen von Interesse, so werden die Methodikbausteine kombiniert. Insofern ist der Prozess von Modularität und Zusammenwirken geprägt, wie die folgenden Beispiele verdeutlichen: Die in Methodik 2 generierten Strecken können als Input für die Manöverdatenbank in Methodik 1 oder als Lastkollektiv für Methodik 3 dienen. Emissionskritische Events oder RDE-Testszenarien aus Methodik 1 lassen sich als Lastkollektive für Reproduktionsgüte von Methodik 3 nutzen. Umgekehrt kann Methodik 3 dazu dienen, den Einfluss nicht korrekt nachgebildeter, thermischer Randbedingungen auf emissionskritische Events oder Tests zu quantifizieren. In einem weiteren Beispiel werden Methodik 2 und 3 in Kombination genutzt, um äußerst emissionskritische und für das AGN-System herausfordernde Prüfläufe zu generieren und damit Anforderungen an die AGN 2025+ simulativ zu untersuchen [10]. Wie zuvor angedeutet erfordert, eine derart ganzheitliche Betrachtung von RDE-Einflüssen ein hohes Maß an Durchgängigkeit, Modularität und Zusammenarbeit.

Ein wichtiger Aspekt, den es zukünftig zu strukturieren gilt, ist eine Übersicht der Messwerte, die für die Validierung der jeweiligen Methodik zu dem spezifischen Anwendungsfall nötig sind. Besonders im Realfahrzeug stellt die Messdatenaufzeichnung eine besondere Herausforderung dar. Jeder Anwendungsfall bedarf spezifischer Messdaten, wie z.B. Ladelufttemperaturen, die teilweise nicht über das Motorsteuergerät zur Verfügung stehen. Einen weiteren Ausblick stellt die Drehmomentenmessung im Fahrzeug dar. 


\section{Literaturverzeichnis}

[1] EUROPÄISCHE KOMMISSION: VERORDNUNG (EU) 2017/1151 DER KOMMISSION - vom 01. Juli 2017 - Überprüfungsdatum 2018-08-22

[2] EUROPÄISCHE KOMMISSION: VERORDNUNG (EU) 2016/427 DER KOMMISSION - vom 10. März 2016 - Überprüfungsdatum 2018-08-22

[3] Raid, B.: Prozess und Methode zur Realisierung fahrzeugnaher dynamischer Versuche am Motorprüfstand. Simulation und Test, Antriebsentwicklung im digitalen Zeitalter, 19. MTZ-Fachtagung, 2017, Hanau.

[4] Maschmeyer, H., Beidl, C., Düser, T. et al.: MTZ - Motortechnische Zeitschrift (2016) 77: 84. https://doi.org/10.1007/s35146-016-0110-8

[5] Maschmeyer, H.; Beidl, C.: Real Driving Emissions Methodology Library for Engine Testbeds. International Symposium on development methodology, 2017, Wiesbaden.

[6] Bauer, S.; Beidl, C.; Düser, T.: Methodik zur Erzeugung RDE-relevanter Szenarien im Prüfstandsumfeld. Simulation und Test, Antriebsentwicklung im digitalen Zeitalter, 19. MTZ-Fachtagung, 2017, Hanau.

[7] Berger, A., Hochmann, G., Mayrhofer, H. et al.: MTZ Extra (2017) 22 (Suppl 1): 26. https://doi.org/10.1007/s41490-017-0002-y

[8] Stefanakis, E.: Elevation Web Services: Limitations and Prospects. GoGeomatics. Magazine of GoGeomatics Canada, November 2015.

[9] Schmidt, D.; Maschmeyer, H.; Beidl, C.; Raß, F.: Neue Verfahren zur effizienten antriebstrangspezifischen RDE-Entwicklung. MTZ-Fachtagung VPC Plus, 2016, Hanau.

[10] Zlojo, H.; Kuznik, A.; Beidl, C.: 2025+ Requirements on Emission Control. Aachener Kolloquium, 2018, Aachen. 